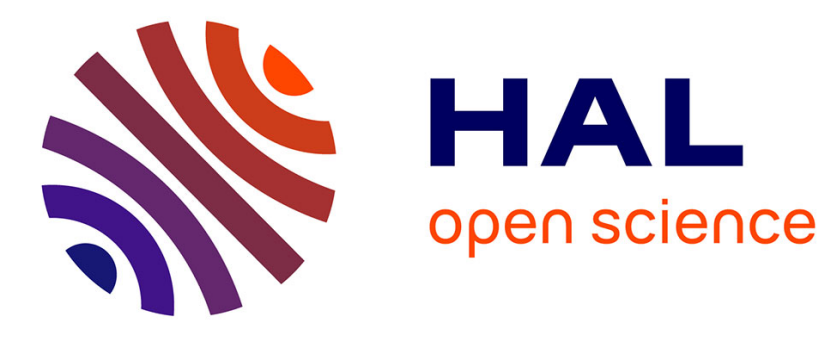

\title{
Luminescences propres et parasites du durène
}

\author{
Y. Meyer, R. Astier
}

\section{To cite this version:}

Y. Meyer, R. Astier. Luminescences propres et parasites du durène. Journal de Physique, 1963, 24

(12), pp.1089-1094. 10.1051/jphys:0196300240120108900 . jpa-00205708

\section{HAL Id: jpa-00205708 https://hal.science/jpa-00205708}

Submitted on 1 Jan 1963

HAL is a multi-disciplinary open access archive for the deposit and dissemination of scientific research documents, whether they are published or not. The documents may come from teaching and research institutions in France or abroad, or from public or private research centers.
L'archive ouverte pluridisciplinaire HAL, est destinée au dépôt et à la diffusion de documents scientifiques de niveau recherche, publiés ou non, émanant des établissements d'enseignement et de recherche français ou étrangers, des laboratoires publics ou privés. 


\title{
LUMINESCENCES PROPRES ET PARASITES DU DURÈNE
}

\author{
Par Y. MEYER et R. ASTIER, \\ Laboratoire de Physique de l'École Polytechnique.
}

\begin{abstract}
Résumé. - On a étudié les luminescences propres et parasites du durène en poudre et en solution et enregistré la phosphorescence T-S de la molécule de durène en solution dans le n-hexane cristallisé à $77^{\circ} \mathrm{K}$. Les raies du spectre obtenu sont fines et on donne une interprétation vibrationnelle basée sur les fréquences $274,723,1274$ et $1580 \mathrm{~cm}^{-1}$. On a observé dans le durène cristallisé l'apparition d'une phosphorescence bleue après action de la température et d'une luminescence verte progressive sous l'effet de l'irradiation. On présente les spectres de ces deux luminescences parasites et on discute les mécanismes correspondants.
\end{abstract}

Abstract. - We have studied the true and parasite luminescences of durene in powder and in solution. The T-S phosphorescence of the durene molecule in solution in crystallised n-hexane at $77 \mathrm{oK}$ has been recorded. The bands of its spectrum are narrow and we give an assessment of its vibrational terms based on the frequencies : 274, 723,1 274 and $1580 \mathrm{~cm}^{-1}$. We have observed that crystallised durene emits a blue phosphorescence after heating and a progressive green luminescence under the action of the U. V. radiation. We give the spectra of these parasite luminescences and discuss the processes involved.

Le monocristal de durène, facile à obtenir, a été utilisé comme solvant pour la luminescence du naphtalène [1], [2] et a été examiné comme éventuel scintillateur [3]. La fluorescence du durène cristallin a été détaillée à très basse température [4], [5]. Dans ces études, aucune difficulté particulière pouvant être attribuée à des impuretés n'a été signalée. Il n'en est cependant pas de même lorsqu'on s'attache à la région du proche ultraviolet et visible où se trouve la phosphorescence $\mathrm{T}$-S du durène.

Kallman et coll. [2] signalent uniquement l'apparition d'une luminescence verte par irradiation du durène cristallisé. Ce phénomène n'a pas été retrouvé par Olness et Sponer [6] qui indiquent par contre l'existence d'un deuxième spectre dans le bleu, de durée de vie plus courte que celle de la phosphorescence propre. Ces différénces sont, à première vue, attribuables à des impuretés. On a alors étudié le durène dans diverses conditions de pureté, d'excitation et de température. On a retrouvé ainsi l'un et l'autre des spectres parasites, établi une relation entre eux et précisé les conditions de leur apparition dans du durène pur. Il a semblé utile de confirmer la distinction entre ces spectres parasites et les émissions de la molécule de durène par l'obtention d'un spectre précis de la phosphorescence propre de cette molécule.

I. Purification. - Les durènes commerciaux cristallisés présentent sous excitation de longueurs d'onde supérieures à $2900 \AA$ (non absorbées par le durène: fig. 1) un grand nombre de bandes de luminescence qu'on peut éliminer partiellement par purification. Les impuretés qui en sont responsables ne peuvent être directement identifiées par absorption ultraviolette, en raison de leur faible concentration.

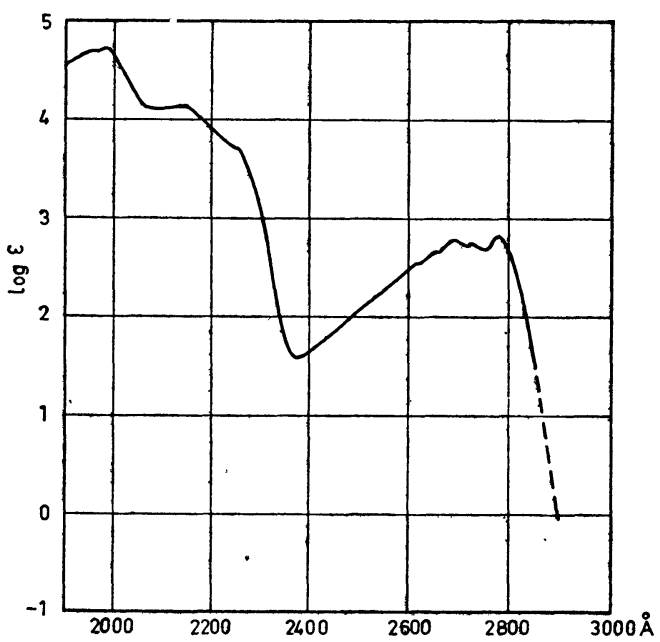

Fig. 1. - Coefficient d'absorption du durène en solution dans l'isooctane à $300^{\circ} \mathrm{K}$ (spectrophotomètre Cary 14).

Après essai de divers procédés de purification, nous avons obtenu une qualité de durène dont la luminescence parasite est extrêmement faible, par cristallisations répétées, en refroidissant jusqu'à $30^{\circ} \mathrm{C}$ une solution dans l'alcool saturée à $60^{\circ} \mathrm{C}$. La limitation à $60^{\circ}$ de la température de dissolution a une importance que la suite expliquera.

II. Dispositif expérimental. - Les spectres d'absorption ont été enregistrés au moyen du spectrophotomètre à double faisceau Cary 14.

Pour la fluorescence, l'excitation est produite par une lampe à vapeur de $\mathrm{Hg}$ haute pression $200 \mathrm{~W}$, 
suivie par un monochromateur à prisme de quartz. L'échantillon se trouve dans une coupelle placée dans un cryostat en contact avec l'azote liquide. Le même spectrophotomètre est utilisé pour l'enregistrement direct des luminescences.

Pour obtenir des spectres de phosphorescence exempts de bandes de fluorescence ou d'excitation, on a utilisé la modulation à $25 \mathrm{~Hz}$ du spectrophotomètre à double faisceau. On déclenche un tube flash au xénon FT 91 (General Electric) placé près de l'échantillon, lorsqu'un signal du spectrophotomètre indique que le PM est masqué pour le faisceau utilisé. Ce signal est prélevé au moyen d'un étage cathodyne à forte impédance d'entrée, puis envoyé sur la grille du thyratron qui commande la décharge d'un condensateur dans le primaire de la bobine HT.

Ce même montage permet de mesurer les durées de vie suffisamment longues. L'enregistreur du Cary 14, commandé par un amplificateur logarithmique, fournit directement la courbe de décroissance de l'intensité lumineuse d'une raie donnée en fonction du temps. Dans le cas d'un déclin exponentiel, il suffit alors de mesurer la pente de la droite obtenue.

III. Luminescences de la molécule de durène. En raison de l'effet bathochrome des groupes méthyl, on peut attendre les niveaux d'énergie de la molécule de durène à quelques centaines de $\mathrm{cm}^{-1}$ de moins que les valeurs connues pour le benzène. C'est en effet ce que l'on a observé en fluorescence comme en phosphorescence.

1) Transition S-S (durène cristallisé). Absorption. - Avec le durène 5 fois recristallisé dans l'alcool, nous avons trouvé dans l'isooctane à $300 \mathrm{oK}$, les coefficients d'absorption (fig. 1) : $\lambda \AA(\log \varepsilon): 2779(2,82) ; 2725(2,75) ; 2690$ $(2,79) ; 2652(2,65) ; 2612(2,53) ; 2255(3,70)$; $2145(4,01) ; 1988(4,71) ; 1966(4,70)$. Le durène cristallisé montre à $300 \mathrm{oK}$ uh spectre peu structuré, tandis qu'à $20^{\circ} \mathrm{K}$ une structure fine apparaît [4]. La bande $0-0$ peut alors être placée à $2792 \AA\left(35818 \mathrm{~cm}^{-1}\right)$.

Fluorescence. - Avec une excitation centrée sur $2750 \AA$, le durène cristallisé donne à $300^{\circ} \mathrm{K}$ un spectre de fluorescence sans structure. Dès $77^{\circ} \mathrm{K}$ une structure très nette apparaît et les principaux maximums que nous avons trouvés correspondent à $4 \AA$ près aux valeurs mesurées à $20^{\circ} \mathrm{K}$. En plaçant la raie $0-0$ à $35790 \mathrm{~cm}^{-1}$, on peut rendre compte de la structure vibrationnelle du spectre à $77 \mathrm{oK}$ au moyen des fréquences 725 et $1280 \mathrm{~cm}^{-1}$. Les autres fréquences trouvées à $20{ }^{\circ} \mathrm{K}$ n'apparaissent pas distinctement dans nos spectres à $77^{\circ} \mathrm{K}$.

2) Transition T-S (durène en solution dans n-hexane). - Peu de données existaient sur la phosphorescence du durène. On n'a trouvé dans la littérature qu'un seul spectre, peu structuré, dont le premier maximum est. à $26500 \mathrm{~cm}^{-1}$ [7]. L'intérêt du choix de certains solvants cristallisés dans l'étude des transitions électroniques a été d'abord mis en évidence par Shpolsky en 1958 [8]. Des raies de luminescence remarquablement fines (de l'ordre de l'angström) ont été par la suite obtenues dans le cas du benzène grâce à l'emploi, comme solvant, du cyclohexane cristallisé à 77 oK [9]. Pour le durène, les raies les plus fines ont été obtenues, à $77 \mathrm{oK}$, dans le $\mathrm{n}$-hexane. Avec une largeur de fente correspondant environ à $2 \AA$ la raie $0-0$ à

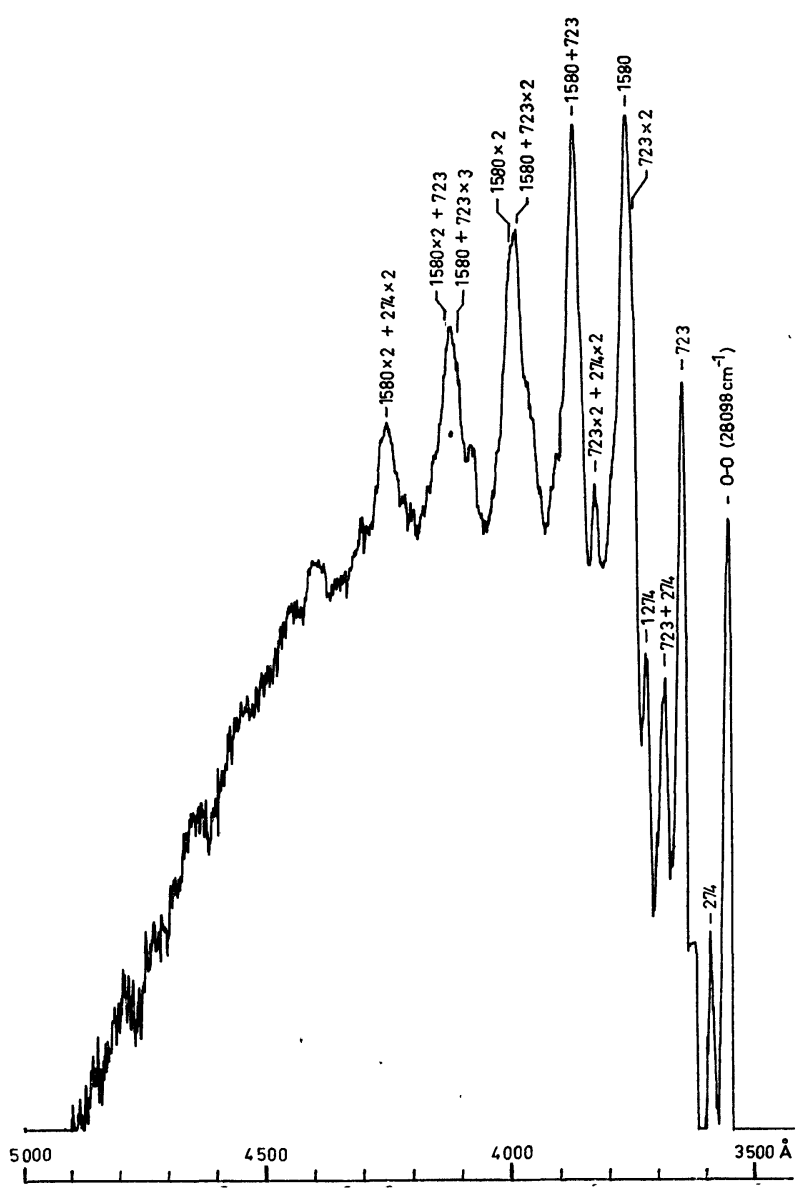

FIG. 2. - Enregistrement (photomultiplicateur 1P 28) de la phosphorescence T-S du durène en solution dans le n-hexane cristallisé à $77 \mathrm{oK}$. Largeur des fentes du monochromateur $=0,2 \mathrm{~mm}$ (soit une largeur spectrale de 6 à $7 \AA$ A). Les fréquences données ont été obtenues avec des fentes de 40 à $60 \mu(\sim 2 \AA)$. Observation : environ $20 \mathrm{~ms}$ après l'éclair d'excitation de durée 0,1 ms.

$28098 \mathrm{~cm}^{-1}$ a une largeur, à mi-hauteur, de $16 \AA$. Le spectre de phosphorescence (fig. 2) a pu ainsi être analysé (tableau I) avec plus de précision que dans un solvant vitreux. Dans l'alcool, la raie 0-0 est située à $27790 \mathrm{~cm}^{-1}$ et la structure est beaucoup moins nette. 
TABLEAU I

Phosphorescence DU DURène en solution DANS LE n-HEXANE GRISTALLISÉ A $77^{\circ} \mathrm{K}$

\begin{tabular}{|c|c|c|}
\hline NT & $\nu\left(\mathrm{cm}^{-1}\right)$ & $\Delta$ \\
\hline $\mathrm{m}$ & 28098 & 0 \\
\hline & 27824 & 274 \\
\hline if & 27579 & 51 \\
\hline $\mathrm{m}$ & 27375 & $\sqrt{2}$ \\
\hline & 27093 & 1005 \\
\hline & 26824 & 1274 \\
\hline ép & 26610 & 1488 \\
\hline$F$ & 26518 & 580 \\
\hline p & 26364 & 1734 \\
\hline p & 26274 & 1824 \\
\hline m & 26082 & 2016 \\
\hline ép & 25913 & 2185 \\
\hline $\mathrm{F}$ & 25793 & 2305 \\
\hline & 25575 & 2523 \\
\hline p & 25368 & 2730 \\
\hline$p$ & 25265 & 2833 \\
\hline p & 252 & \\
\hline & 25056 & 3042 \\
\hline$F$ & 24963 & 3135 \\
\hline ép & 24845 & 3253 \\
\hline & 6 & 00 \\
\hline$p$ & 140 & 3761 \\
\hline$\Gamma$ & 4213 & 3885 \\
\hline & 24004 & 4094 \\
\hline Min & 23469 & 4629 \\
\hline & 23180 & 491 \\
\hline
\end{tabular}

que nous avons obtenues en phosphorescence. L'interprétation du spectre, donné ci-dessus, met en évidence quatre fréquences essentielles : 274,723 , 1274 et $1580 \mathrm{~cm}^{-1}$. La première et la dernière sout nouvelles et n'apparaissent pas en fluorescence. Par contre, la fréquence trouvée à $475 \mathrm{~cm}^{-1}$ par McClure [4] et à $508 \mathrm{~cm}^{-1}$ par Benarroche [5] n'apparaît pas en phosphorescence.

IV. Spectres de luminescence parasite observés dans le durène cristallisé. - Les luminescences parasites sont celles que l'on peut éliminer par purification. Dans le cas du durène pur, deux luminescences peuvent réapparaître facilement :

10 Une phosphorescence bleue de durée de vie de l'ordre de 0,1 à $0,01 \mathrm{~s}$, visible à $77^{\circ} \mathrm{K}$ et non à $300 \mathrm{oK}$, très bien structurée (fig. 3). L'analyse

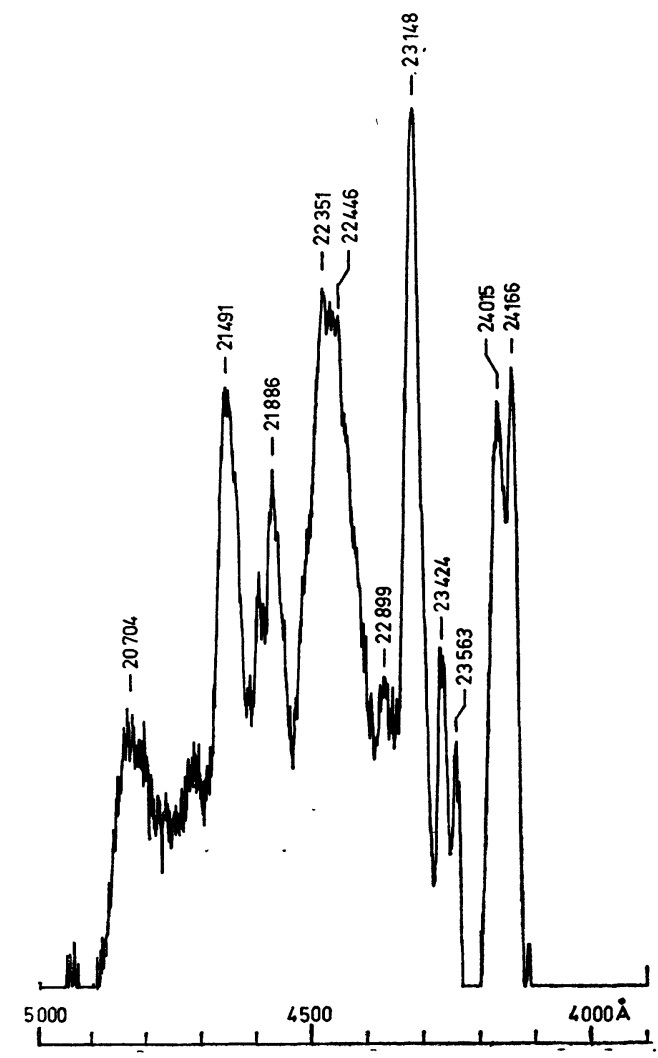

FIg. 3. - Enregistrement (photomultiplicateur 1P 28) de la phosphorescence parasite à $770 \mathrm{~K}$ du durène cristallisé ayant été chauffé, observée environ $20 \mathrm{~ms}$ après l'éclair d'excitation.

vibrationnelle de ce spectre laisse penser qu'il pourrait être en réalité composite et formé de bandes dues à plusieurs molécules de structures voisines. Cette phosphorescence correspond bien à la partie à déclin rapide de l'émission trouvée par Olness et Sponer [6] et confirme ainsi leur hypothèse d'une impureté. tement. Les principales sont rassemblées dans le tableau II (p. 1092) et confrontées avec les valeurs
3) Comparaison des fréquences du durène DANS L'ÉTAT FONDAMENTAL. - Les valeurs publiées des fréquences de vibration du durène qui ont été obtenues par effet Raman, par absorption infrarouge et par fluorescence, ne concordent pas exac- 
TABLEAU II

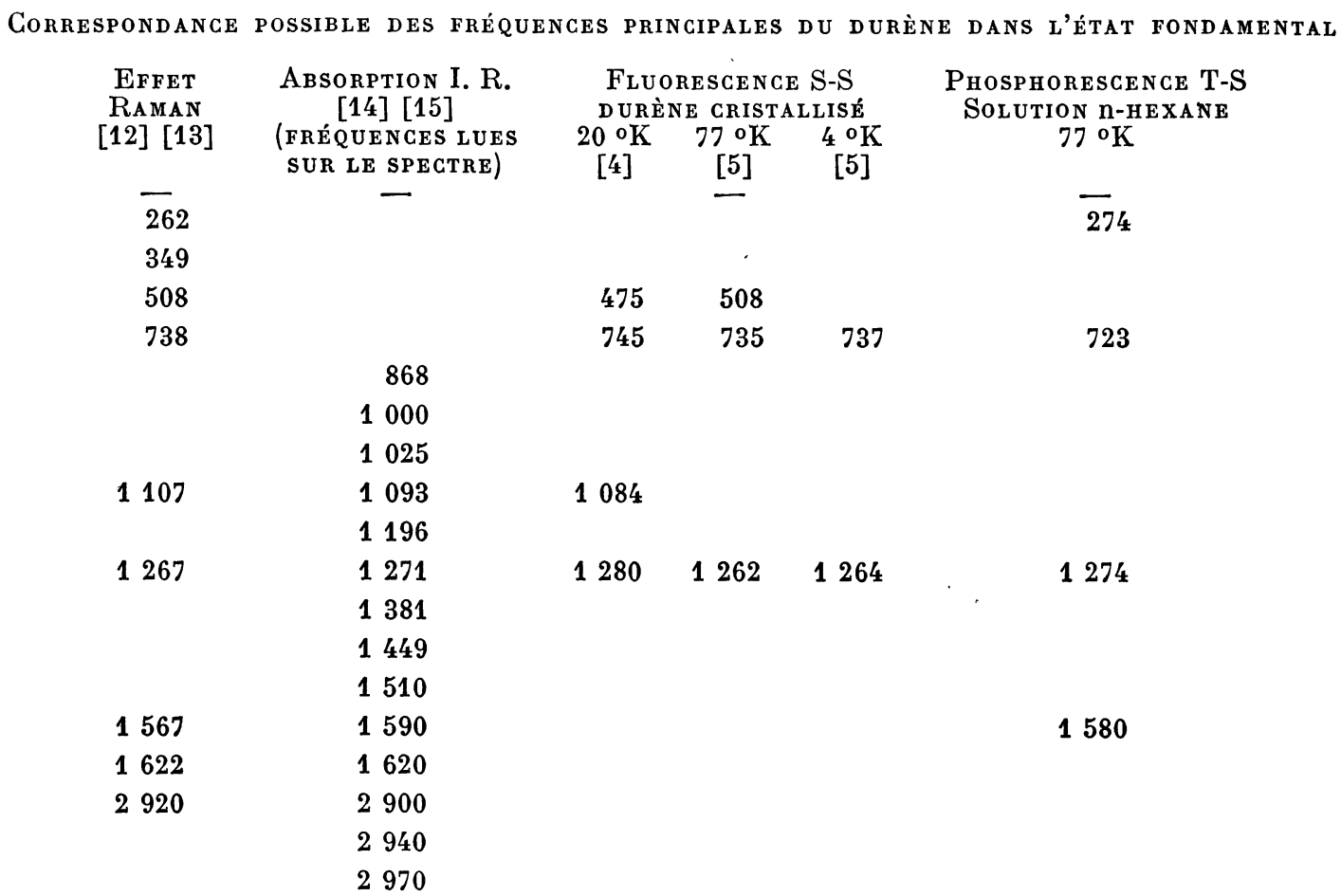

L'élimination des impuretés responsables demande des précautions. La fusion par zone conduit à un produit, qui, examiné à $77^{\circ} \mathrm{K}$, sous excitation de $3100 \AA$ montre des zones phosphorescentes inégalement réparties. La recristallisation décrite plus haut donne au contraire, un produit régulièrement exempt de phosphorescence bleue. Pour expliquer ces différences, on a vérifié qu'un chauffage léger, même sous vide, accroît considérablement cette phosphorescence. En procédant sur un durène purifié, puis fondu pendant $10 \mathrm{mn}$ à $100^{\circ} \mathrm{C}$ à une recristallisation partielle, on sépare une solution qui fournit après concentration une nouvelle bande d'absorption à $3450 \AA$ ( $f i g$. 4). Cette bande correspond à la région d'excitation de la phosphorescence. On n'a pas trouvé de fluorescence associée : celle-ci devrait débuter entre 3000 et $3800 \AA$. Son absence s'explique si l'on admet une oxydation (à partir de l'oxygène fixé sur le durène) conduisant à des aldéhydes, cétones ou quinones : ces composés sont caractérisés par la forte conversion de $\mathrm{S}_{1}$ vers $\mathrm{T}_{1}$ qui empêche l'apparition de la fluorescence.

En maintenant à $100{ }^{\circ} \mathrm{C}$ une série de tubes scellés sous vide, contenant du durène cristallisé, on a constaté que si la phosphorescence du premier tube (fondu $3 \mathrm{mn}$ ) est beaucoup plus intense que celle du produit initial, celle des autres tubes n'augmente pas proportionnellement avec le temps et atteint un palier au bout de 3 heures. Ce résultat peut s'expliquer si le durène obtenu par recristal-

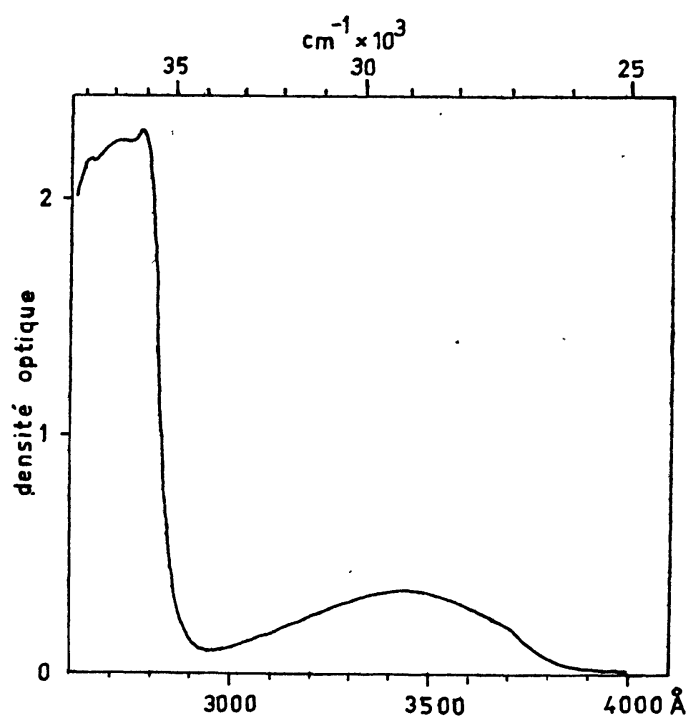

FIg. 4. - Absorption d'une solution dans l'alcool à $300 \% \mathrm{~K}$, de durène ayant été fondu. Une partie du durène a été éliminée par recristallisation, pour augmenter la concentration des impuretés. On voit effectivement apparaître une nouvelle bande vers $3450 \AA$.

lisation renferme encore une petite quantité d'oxygène, susceptible de se combiner lentement à lui. Enfin, on a constaté que la combinaison a lieu même à température ambiante : au bout de quelques semaines, les paillettes de durène pur sont à nouveau faiblement phosphorescentes. 
$2^{\circ}$ Une LUMINESCENGE Verte qui apparaît et augmente d'intensité avec la durée de l'irradiation. Elle est visible à $300 \mathrm{oK}$ comme à $77^{\circ} \mathrm{K}$, mais ne passe pas à travers le phosphoroscope, elle est assez peu structurée ( $f i g .5)$. Ce phénomène a déjà été

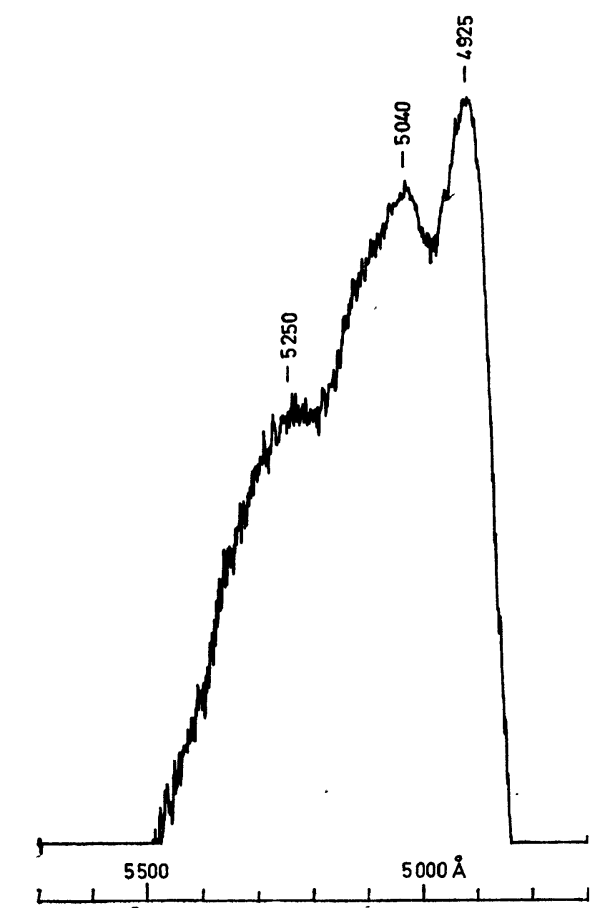

FIG. 5. - Enregistrement photoélectrique (PM 1P 28) de la luminescence verte progressive du durène cristallisé à $300^{\circ} \mathrm{K}$. Fente $0,2 \mathrm{~mm}(\sim 7 \AA)$.

signalé par Kallmann [2] sous l'action des rayons $\mathrm{X}$ ou $\gamma$ et de la raie $2537 \AA \mathrm{du} \mathrm{Hg}$. Le spectre donné correspond à celui que nous avons observé. Cependant, la disparition de cette luminescence n'a pu être mise en évidence dans les temps indiqués, et elle est produite et excitée par des radiations de plus grande $\lambda$ (au moins jusqu'à $3340 \AA$ ) si le durène excité contient les impuretés à phosphorescence bleue. La vitesse d'apparition de la luminescence verte est liée à l'intensité de la phosphorescence bleue (fig. 6). L'apparition de la luminescence est plus rapide à $300^{\circ} \mathrm{K}$ qu'à $77^{\circ} \mathrm{K}$. Enfin, elle apparaît sur toute la trace du faisceau d'excitation $(\sim 3100 \AA)$ à l'intérieur d'un monocristal.

C'est un cas de "phosphorescence progressive " selon l'expression de Goldstein. Il peut, en fait, s'agir de plusieurs mécanismes distincts. Toutefois, l'hypothèse la plus simple : photo-oxydation ou photodissociation de l'impureté à phosphorescence bleue (possible avec ces énergies [11]) qui paraît à première vue devoir être écartée, peut être maintenue si on admet l'existence d'un transfert d'énergie entre l'impureté à phosphorescence bleue qui absorbe et le composé à luminescence verte qui émet. L'apparition de cette luminescence devrait être accompagnée de l'apparition d'une absorption située dans le visible, ce qui n'est pas le cas. (La coloration jaunâtre que prend le durène irradié par $\operatorname{des} \lambda<3600 \AA$ n'est pas liée à la luminescence verte, car cette dernière n'est pas excitée par des raies

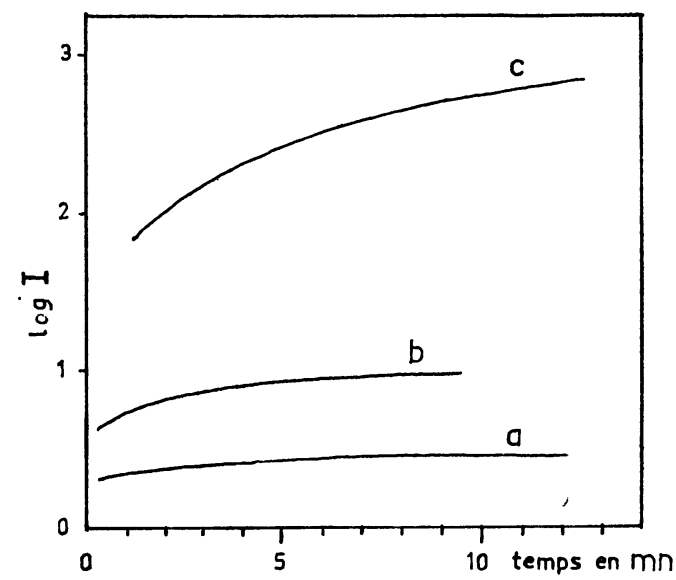

Fig. 6. - Apparition de la luminescence verte parasite du durène cristallisé, mesurée dans la bande $4935 \AA$ à 300 \% $\mathrm{K}$.

a) Durène purifié.

b) Durène purifié, puis fondu.

c) Durène présentant une phosphorescence bleue intence.

visibles de la lampe à vapeur de mercure.) Toutefois, même si la concentration d'impureté produite par l'ultraviolet est trop faible pour être détectée par absorption, la luminescence peut être importante si elle est alimentée par le transfert d'énergie.

Il est vraisemblable que le cristal joue un rôle dans ce transfert car une simple dissolution du durène irradié, suivie d'une recristallisation, suffit pour faire disparaittre la luminescence verte. On peut donc envisager, comme l'a suggéré Kallmann, la formation de centres luminogènes. Mais il est alors surprenant que la même luminescence verte apparaisse aussi bien par action de radiations absorbées uniquement par l'impureté responsable de la phosphorescence bleue que par action de radiations"(rayons $\mathrm{X}$ et $\gamma$ et raie $2537 \AA \mathrm{du} \mathrm{Hg}$ ) absorbées uniquement par le durène [2]. On peut penser que la fluorescence ultraviolette du durène luimême est suffisante, après un temps assez long, pour irradier suffisamment les molécules de l'impureté à phosphorescence bleue toujours présentes sous forme de traces dans le durène le plus " pur ».

Nous tenons à remercier $M$. Teissier du Cros pour les conseils qu'il a bien voulu nous donner. Nous remercions également $M$. Rajic qui a mis au point le montage électronique et M. Verron pour la préparation du durène pur.

Manuscrit reçu le 31 juillet 1963. 


\section{BIBLIOGRAPHIE}

[1] MaCuune (D. S.), J. Chem. Physics, 1954, 22, 16681675.

[2] Kallmann (H.), Hayakawa (S.) et Magnante (P.), "Luminescence of organic and inorganic materials ", p. 244 (Intern. Conf. New-York Univ., éd. KallmannSpruch).

[3] SangSter (R. C.) et Irvine (J. W.), J. Chem. Physics, 1956, 24, 670-715.

[4] Schnepp (O.) et McGlure (D. S.), J. Chem. Physics, $1959,30,874-878$.

[5] Benarroche (M.), C. R. Acad. Sc., 1962, 254, 459-461.

[6] Olness (D.) et Sponer (H.), J. Chem. Physics, 1963, 38, 1779-1782.

[7] Czekalla (J.), Briegleb (G.), Herre (W.) et VahlenSIEGK (H. J.), Z. Elektrochem., 1959, 63, 715-721.
[8] Shpolsky (E. V.), Optika I. Spect., 1958, 4, 620.

[9] Courpron (C.), Lochet (R.), Meyer (Y.) et Rousset (A.), C. R. Acad. Sc., 1960, 250, 3095-3097.

[10] Dikoune (P. D.), Petrov (A. A.) et Svechnikov (B. J.), Zhur Eksptl. Toret. Fiz., 1951, 21, 150-163.

[11] LEAGH (S.), "Luminescence of organic and inorganic materials ", p. 177.

[12] Kolhrausch (K. W.) et Pongratz (A.), Wiener Ber. Kl II b, 1934, 143, 358.

[13] Ale kSAnyan et coll., Izvest. Akad. Nauk SSSR Ser. Fiz., 1955, 19, 225-233.

[14] Sadtler Standard Spectra, midget edition, 1959, courbe no 13386.

[15] Cannon (C. G.) et Sutherland (G. B. B. M.), Spectrochim. Acta, 1951, 4, 373-395. 